http://jmscr.igmpublication.org/home/

ISSN (e)-2347-176x ISSN (p) 2455-0450

crossref DOI: https://dx.doi.org/10.18535/jmscr/v7i10.100

Journal Of Medical Science And Clinical Research

IGM Publication

An Official Publication of IGM Publication

\title{
Isolation and Antimicrobial Susceptibility Pattern of Klebsiella Pneumoniae in sputum sample with Lower Respiratory Tract infection suspected patients at a Tertiary Care Hospital Rajasthan
}

\author{
Authors \\ Farkhanda ${ }^{1}$, Rachna $^{2}$, Bhawani Shankar Verma ${ }^{3} *$, Prasanna Gupta ${ }^{4}$ \\ Department of Microbiology, National Institute of Medical Science and Research (NIMSR), Jaipur \\ *Corresponding Author \\ Bhawani Shankar Verma \\ Department of Microbiology, National Institute of Medical Science and Research (NIMSR), Jaipur
}

\begin{abstract}
Background: Gram-negative pathogens are an important cause of community and hospital-acquired infections throughout the world. Klebsiella pneumoniae has become one of the more common causes of these infections and one of the important aspects of Klebsiella associated infections is the emergence of multi-drug resistant strains particularly those involved in nosocomial infections or hospital settings. This study was done to determine the isolation rate of Klebsiella, their antibiogram and the presence of resistant strains from sputum samples.
\end{abstract}

Aims and Objective: Isolation and Antimicrobial Susceptibility Pattern of Klebsiella Pneumoniae in a sputum sample with Lower Respiratory Tract infection suspected patients that are study were undertaken to detect the incidence of $K$. Pneumoniae isolated from different clinical specimens in the Department of Microbiology in NIMS Medical College Jaipur.

Materials \& Methods: A total number of 270 sputum samples were included in the 6-month study. Klebsiella was identified by standard microbiological techniques and antibiotic susceptibility testing was done by Kirby-Bauer disc diffusion method and interpreted as per CLSI guidelines.

Results: A total of 270 cultures from sputum samples and a total of 41 Klebsiella pneumoniae were isolated thus culture positivity was $35.65 \%$, and other organisms were $64.35 \%$. A total of Klebsiella pneumonia isolates, 25 (60.9\%) were from males and 16 (39.1\%) were from females. Isolation rate was highest in the male patient then female patients were low rate found in this study. The more sensitive to Amikacin (90.24\%), Meropenem (85.35\%), Imipenem (80.48\%). Isolates showed high resistance to Ceftriaxone (73.17\%), Levofloxacin (70.73\%), and Aztreonam (52.9\%) and Ampicillin (100\%) because it is intrinsic resistance.

Conclusion: The data of this study may be used to determine trends in antimicrobial susceptibilities to formulate local antibiotic policies and overall to assist clinicians in the rational choice of antibiotic therapy.

Keywords: Klebsiella pneumoniae, Antibiotic resistance, Antibiotic susceptibility testing, Antibiotic policy.

\section{Introduction}

Lower respiratory tract infections are among the most common infectious diseases of humans worldwide. Klebsiella pneumoniae has become one of the most common causes of these respiratory tract infections. Currently, drug 
resistance to human pathogenic bacteria is frequently being reported worldwide ${ }^{[1]}$. Microorganisms develop resistant to both older and newer antibiotics. Bacteria are known to have an increasing ability to transmit and acquire resistance to these therapeutic drugs and also transferring the resistance from one bacteria to other resistant bacteria poses a threat to public health in such a way thereby increasing morbidity and mortality ${ }^{[2]}$.

Antibiotics were considered to be the most effective therapeutic drugs to combat microbial infections. But as a result of the overuse, these have been an emergence and spread of multidrugresistant most strains of Enterobacteriaceae among different groups of microorganisms. Infections resistant bacteria are emerging threats all over the world both as hospital-acquired as well as community-acquired microorganisms ${ }^{[3]}$.

Klebsiella is a Gram-negative, non-motile, capsulated, lactose fermenting, facultative anaerobe belonging to the Enterobacteriaceae family. It is the second most popular member of the aerobic bacterial flora of the human intestine. It is the most common causative agent of nosocomial and community-acquired infections. It is responsible for severe lobar pneumoniae. In both community and hospital settings resistant bacteria are emerging as a worldwide threat even in common infections. It is known to produce community-acquired pneumoniae especially in chronic alcoholics, urinary tract infection, wound infections, blood infections and infections in the intensive care unit ${ }^{[4]}$. Though $K$. pneumoniae is a commensal of the human intestine it can cause bronchopneumonia, ventilator-associated pneumonia in hospitalized patients, urinary tract infections, postoperative wound infections, bloodstream infections, etc ${ }^{[5]}$.

Biochemically typical strains of Klebsiella pneumoniae are resistant to a wider range of antibiotics than are most Escherichia coli strains. They are nearly always naturally resistant to Ampicillin ${ }^{[6]}$

\section{Material and Methods}

Total of 270 sputum samples was collected and Klebsiella pneumoniae were identified by standard microbiological techniques and antibiotic susceptibility testing was done by Kirby-Bauer disc diffusion method and interpreted as per CLSI guidelines 2019 in the Department of NIMS Medical College Jaipur.

Sample Collection and Culture: Sputum samples are collected in a sterile container and Sputum samples were aseptically inoculated on to Blood agar, Chocolate agar and MacConkey agar plates and incubated overnight at $37^{\circ} \mathrm{C}$ temperature. Klebsiella pneumoniae isolates were identified by their morphology and biochemical characteristics. Morphology of Klebsiella identified were large dome-shaped colonies on Blood and Chocolate agar and lactose fermenting mucoid colonies on MacConkey agar \& Antibiotic susceptibility test on Muller- Hinton agar plate. In Gram staining, Gram-negative, short, blunt rods were seen. The biochemical characters identified were, Indole test-negative, Methyl red test-negative, VogesProskauer test-positive, Citrate utilization testpositive Urease test-Positive and Triple sugar iron-acid and abundant gas production from glucose, lactose, sucrose, and maltose, mannitol sugar fermentation tests ${ }^{[7]}$.

Antibiotic susceptibility testing: The antimicrobial sensitivity of the test strains of Gram Negative antibacterial drugs was done using the KirbyBauer disk diffusion method ${ }^{[8]}$. The commercial available antibiotic discs used for the study were Meropenem $(10 \mu \mathrm{g})$, Imipenem $(10 \mu \mathrm{g})$, Amikacin $(30 \mu \mathrm{g})$, Ceftriaxone $(30 \mu \mathrm{g})$, Levofloxacin $(5 \mu \mathrm{g})$, Co-trimoxazole $(10 \mu \mathrm{g})$, Ampicillin $(10 \mu \mathrm{g})$, Gentamycin (10 $\mu \mathrm{g})$, Ceftazidime $(30 \mu \mathrm{g})$, Ciprofloxacin $(5 \mu \mathrm{g})$, Tetracycline $(30 \mu \mathrm{g})$,

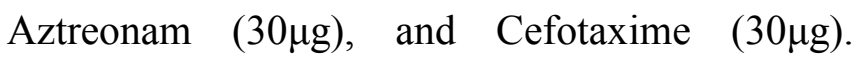
Antimicrobial susceptibility of Klebsiella pneumoniae to different antibiotics is used and treat for pneumoniae infections. From antimicrobial susceptibility, and results are interpreted, considering the antimicrobial 
susceptibility probable drug of choice is selected for Klebsiella Pneumoniae ${ }^{[9]}$.

\section{Results}

During the 6 months, a total of 270 sputum samples were processed for culture and sensitivity testing. Sputum samples of patients of all age groups and both sexes were processed. A total of 41 Klebsiella pneumoniae were isolated thus culture positivity was $35.65 \%$, and other organisms were $64.35 \%$ (Fig.1). A total of Klebsiella pneumonia isolates, $25(60.9 \%)$ were from males and $16(39.1 \%)$ were from females (Fig.2). Isolation rate was highest in the male patient then female patients were low rate found in this study, followed by above 60 years of males (48.0\%) and 46-60 years females $(37.5 \%)$ in this age group. The lowest rate of isolation was observed in males (4.0\%) and females (6.2\%) aged 0 - 15 years (Table No.1 \& Fig.3).

The more sensitive to Amikacin (90.24\%), Meropenem (85.35\%), and Imipenem (80.48\%). Isolates showed high resistance to Ceftriaxone (73.17\%), Levofloxacin (70.73\%), Aztreonam (52.9\%) and Ampicillin (100\%) because it is naturally resistance for Klebsiella spp. (Table No. 2 \& Fig.4).. The present study reveals the frequency of isolation of Klebsiella pneumonia from sputum samples and their tendency towards antibiotic resistance.

Fig.1: Distribution of Klebsiella pneumonia and other organism

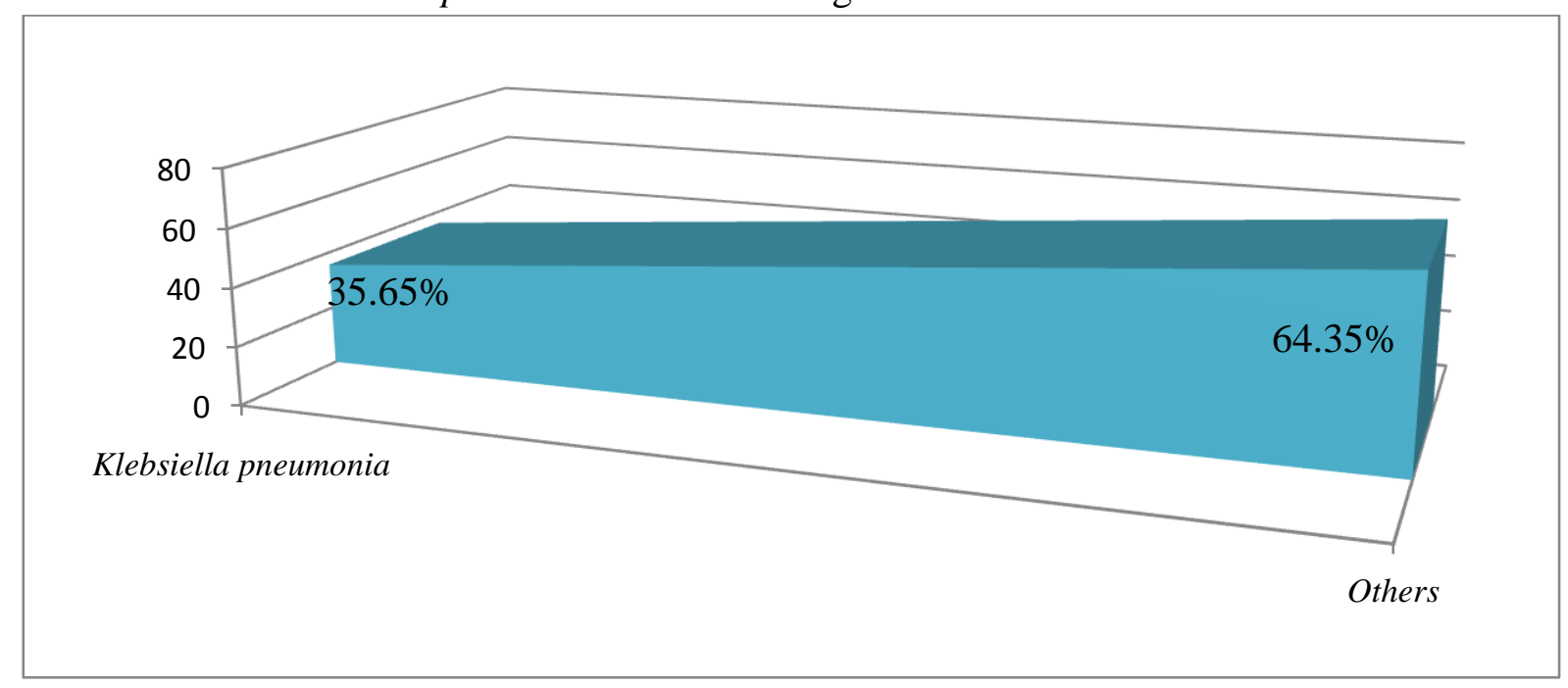

Fig.2: Distribution of sex wise

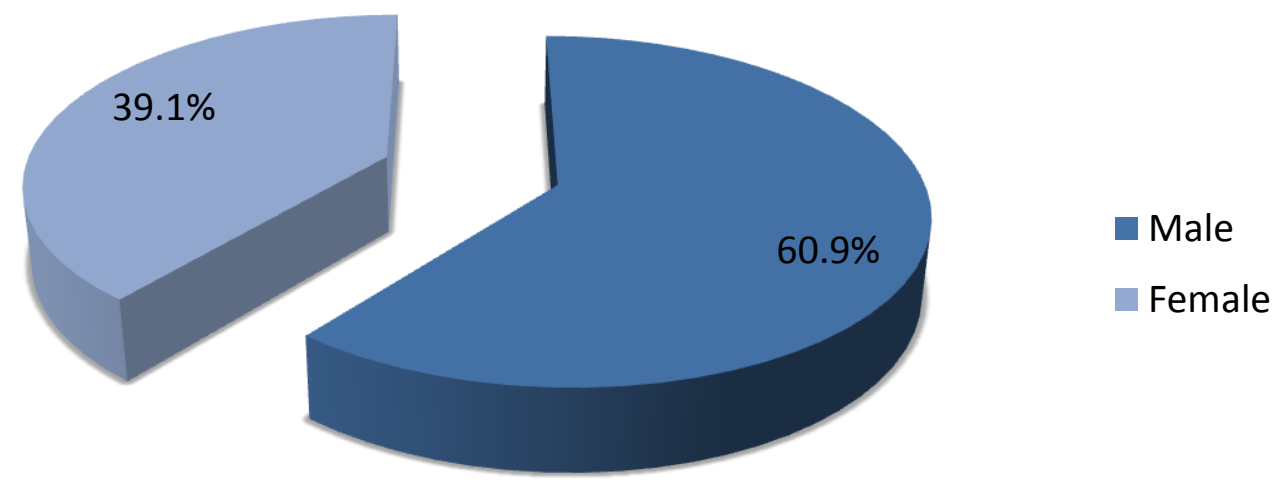


Table no. 1: Distribution of age group

\begin{tabular}{|l|c|c|}
\hline Age & Male $\mathrm{n}=25(\%)$ & Female $\mathrm{n}=16(\%)$ \\
\hline $0-15$ & $1(4.0)$ & $1(6.2)$ \\
\hline $16-30$ & $2(8.0)$ & $2(12.5)$ \\
\hline $31-45$ & $3(12.0)$ & $2(12.5)$ \\
\hline $46-60$ & $7(28.0)$ & $6(37.5)$ \\
\hline $60-$ Above & $12(48.0)$ & $5(31.3)$ \\
\hline
\end{tabular}

Fig. 3: Distribution of age group wise (\%)

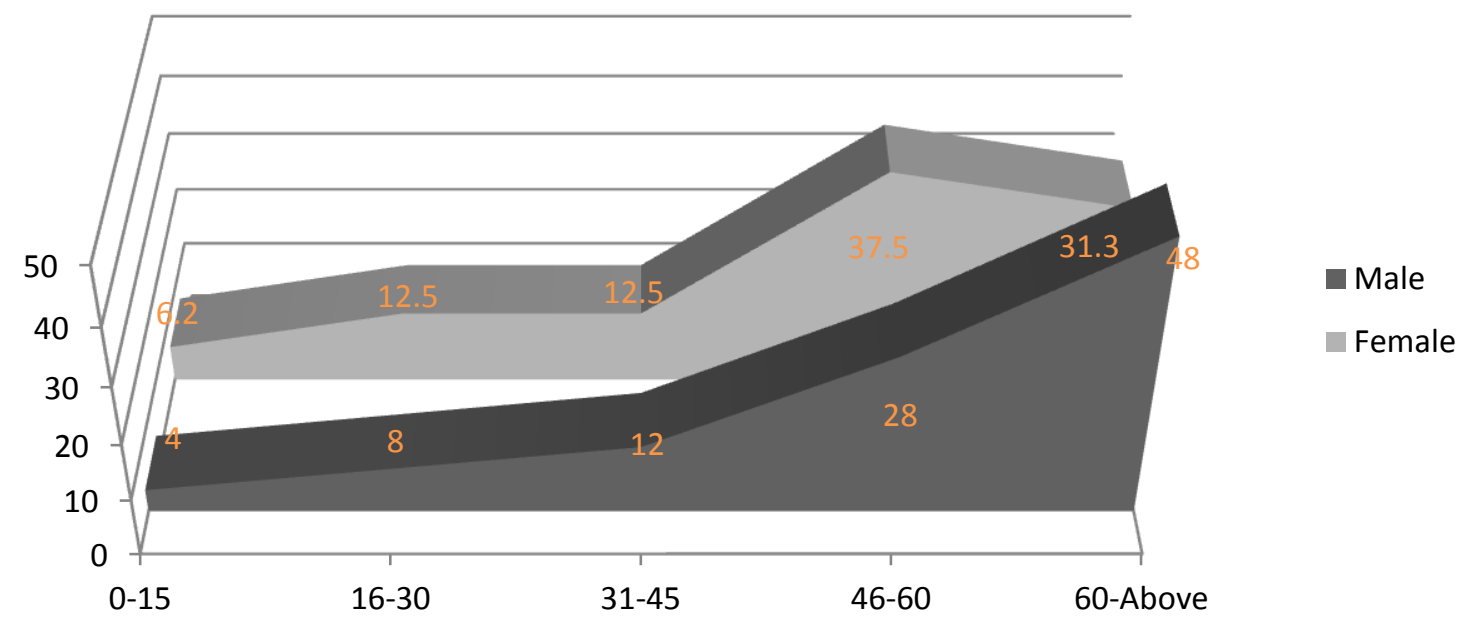

Table No. 2: Distribution of antibiotic susceptibility pattern

\begin{tabular}{|l|c|c|c|}
\hline S.N. & Antibiotics & Sensitive $(\mathrm{n}=41)$ & Resistant $(\mathrm{n}=41)$ \\
\hline 1 & Amikacin $(30 \mu \mathrm{g})$ & $37(90.24 \%)$ & $4(9.76 \%)$ \\
\hline 2 & Meropenem $(10 \mu \mathrm{g})$ & $35(85.36 \%)$ & $6(14.64 \%)$ \\
\hline 3 & Imipenem $(10 \mu \mathrm{g})$ & $33(80.48 \%)$ & $8(19.52 \%)$ \\
\hline 4 & Piperacillin- Tazo. $(100 / 10 \mu \mathrm{g})$ & $29(70.73 \%)$ & $12(29.27 \%)$ \\
\hline 5 & Ceftazidime $(30 \mu \mathrm{g})$ & $21(51.22 \%)$ & $20(48.78 \%)$ \\
\hline 6 & Ciprofloxacin $(5 \mu \mathrm{g})$ & $20(48.78 \%)$ & $21(51.22 \%)$ \\
\hline 7 & Cotrimoxazole $(10 \mu \mathrm{g})$ & $19(46.34 \%)$ & $22(53.66 \%)$ \\
\hline 8 & Gentamycin $(10 \mu \mathrm{g})$ & $18(43.90 \%)$ & $23(56.10 \%)$ \\
\hline 9 & Tetracycline $(30 \mu \mathrm{g})$ & $18(43.90 \%)$ & $23(56.10 \%)$ \\
\hline 10 & Cefotaxime $(30 \mu \mathrm{g})$ & $16(39.02 \%)$ & $25(60.98 \%)$ \\
\hline 11 & Aztreonam $(30 \mu \mathrm{g})$ & $14(34.15 \%)$ & $27(65.85 \%)$ \\
\hline 12 & Levofloxacin $(5 \mu \mathrm{g})$ & $12(29.27 \%)$ & $29(70.73 \%)$ \\
\hline 13 & Ceftriaxone $(30 \mu \mathrm{g})$ & $11(26.83 \%)$ & $30(73.17 \%)$ \\
\hline 14 & Ampicillin $(10 \mu \mathrm{g})$ & 00 & $41(100 \%)$ \\
\hline
\end{tabular}


Fig.4: Distribution of antibiotic susceptibility pattern

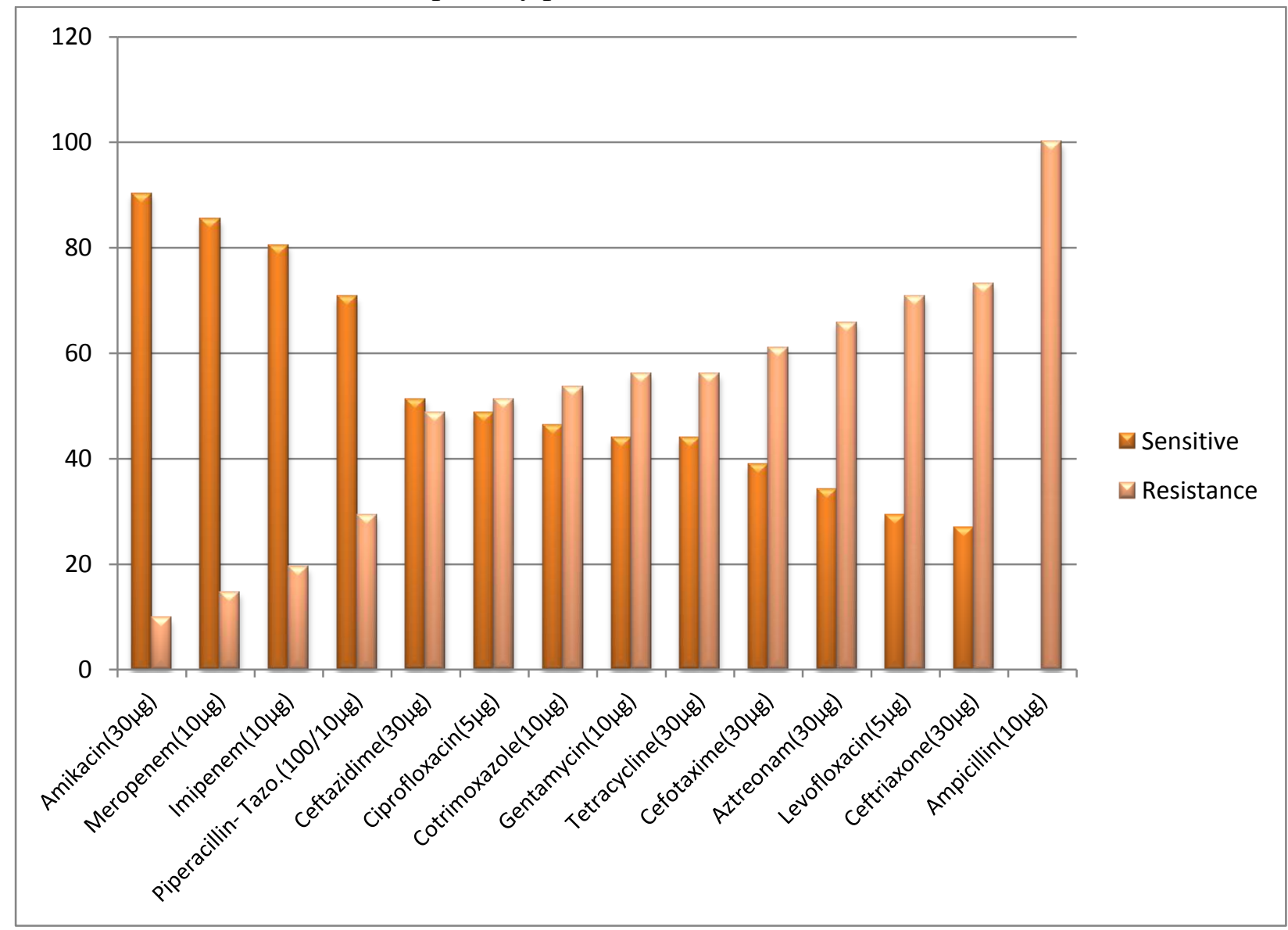

\section{Discussion}

The present study reveals that, total 41 Klebsiella pneumoniae were isolated thus culture positivity was $35.65 \%$, and other organisms were $64.35 \%$ and Klebsiella pneumoniae infection was predominant organism isolated from the age-wise distribution of the clinical isolates of Klebsiella pneumoniae showed that most of $(60.9 \%)$ were from males and the patients were from the elderly age group. 16 (39.1\%) were from females Klebsiella infection was more commonly seen in persons aged above $60 \mathrm{yrs}$. age ${ }^{[10]}$.

Male dominance of infection was observed in the age group above $60 \%$ years $(48 \%), 45-60$ years $(28 \%)$ and $31-45$ years $(12 \%)$ than females $(12.5 \%, 31.3 \%$, and $37.5 \%$ respectively). The higher infection in males of $>60$ years was mainly associated with chronic obstructive pulmonary diseases (COPD), pneumonia and other respiratory diseases. Its compare to Shilpa $\mathrm{K}$ et al (2016) its results similar to our study ${ }^{[1]}$.
Our find out 51 of more Amikacin (90.24\%) and Carbapenems such as Meropenem (85.36\%), and Imipenem (80.48\%) were the most sensitive antibiotic against Klebsiella pneumoniae and compare between Shah RK et al and Shilpa K et $a l^{[12]}$.

Significant high resistance to Ampicillin (100\%), Ceftriaxone (73.17\%), and Levofloxacin (70.73\%) there are reports covering high levels of resistance of Klebsiella Pneumoniae and Ampicillin is naturally resistance to $\mathrm{K}$. pneumonia. This study is compared to Okonko I.O.et al and Shilpa $\mathrm{K}$ et $a l^{[13]}$

This may be due to the production of $\beta$-lactamase enzymes which cause the hydrolysis of the $\beta$ lactam ring resulting in inactivation of $\beta$-lactam antibiotics such as 3rd generation cephalosporins $^{[14]}$.

The $\beta$-lactam antibiotics dose, as well as the incidence of toxicity, subsequently reduced if beta-lactamase inhibitors are used $\mathrm{it}^{[15]}$. Another mechanism is associated with penicillin-binding 
protein 2a (PBP2a), encoded by mecA2. Another gene involved in Penicillin resistance in Staphylococci is Blaz which encodes $\beta$ lactamase $^{[16]}$.

\section{Conclusion}

In conclusion, high antibiotic resistance of Klebsiella pneumoniae towards commonly employed antibiotics are the major reasons for prolonged infections, increased hospitalization, increased cost of therapy and enhanced morbidity and mortality rates.

$K$. pneumoniae infection was predominant in males, 45-60 years and above 60 years of age. The common predisposing factors associated with Klebsiella pneumonia infection were chronic obstructive pulmonary diseases (COPD), pneumonia and other respiratory diseases. $K$. pneumoniae was found to be most sensitive to Amikacin, Meropenem, Imipenem, PiperacillinTazobactam, Ciprofloxacin, and Ceftazidime. Considering the antibiogram, Amikacin, Meropenem, Imipenem, and PiperacillinTazobactam should be preferred drugs for $K$. pneumoniae infection isolated from sputum. Regular surveillance of antibiotic susceptibility patterns may help to overcome the rash use of antibiotics a major cause of the emergence of drug resistance among $K$. pneumoniae and to develop antibiotic policies. The data of this study may be used to determine trends in antimicrobial susceptibilities to formulate local antibiotic policies and overall to assist clinicians in the antibiotic therapy patterns.

\section{Reference}

1. Podschun, R., Ullmann, U. 1998. Klebsiella spp. As nosocomial pathogens: epidemiology, taxonomy, typing methods, and pathogenicity factors. Clin. Microbiol. Rev., 11(4): 589-603.

2. Steinmann J, Kaase M, Gatermann S, Popp W, Steinmann E, Damman M, Paul A, Saner F, Buer J, Rath P M; Outbreak due to Klebsiella pneumonia strain harbouring
KPC-2 and VIM-1 in a German university hospital. Euro Surveill 2011; 16(33): 19944.

3. Chaudhary B.L., Srivastava Shailja, Singh Brij Nandan and Shukla Snehanshu; Nosocomial Infection due to Multidrug Resistant (MDR) Escherichia coli and Klebsiella pneumoniae in Intensive Care Unit. International Journal of Current Microbiology and Applied Sciences 2014; 3(8):630-635.

4. Sikarwar Archana Singh and Batra Harsh Vardhan; Prevalence of Antimicrobial Drug Resistance of Klebsiella pneumoniae in India. International Journal of Bioscience, Biochemistry and Bioinformatics 2011; 3(1): 211-215.

5. Paterson DL, Bonomo RA. Extendedspectrum beta- lactamases: A clinical update. Clin Microbiol Rev 2005;18: 657- 86.

6. Patrick R Murray, Barry Holmes, Hazel M. Aucken. 2005. Topley \& Wilson s Microbiology \& Microbial Infections. Volume 2. 10th edition. Salisbury, UK: Edward Arnold Ltd.

7. K.N.Ravichitra, P.Hema Prakash, S. Subbarayudu and U. Sreenivasa Rao; Isolation and antibiotic sensitivity of Klebsiella pneumonia from pus, sputum and urine samples. Int. J. Curr. Microbiol. App. Sci 2014; 3(3): 115-119.

8. Bauer, AW., Kirby, WMM., Sherris, JC., and Turck, M. 1966. Antibiotic susceptibility testing by a standardized single disk method. Am. J. Clin. Pathol., 45: 493-496.

9. Asati Rakesh Kumar; Antimicrobial Sensitivity Pattern of Klebsiella Pneumoniae isolated from Sputum from Tertiary Care Hospital, Surendra nagar, Gujarat and Issues Related to the Rational Selection of Antimicrobials. Scholars Journal of Applied Medical Sciences (SJAMS) 2013; 1(6):928-933. 
10. Shashidhar Vishwanath, Kiran Chawla, Anusha Gopinathan; Multidrug resistant Gram-negative bacilli in lower respiratory tract infectons. Iranian Journal of Microbiology 2013; 4(5):323-327.

11. Osazuwa F, 1Mordi RM, Osazuwa E, Taiwo SS, Alli OAT, Ogbolu DO, Akanni EO, Anukam; Klebsiella has taken lead among uropathogens in University of Benin Teaching Hospital, Benin City, Nigeria-An observation. New York Science Journal. 2010; 3(11):61-64.

12. Shah RK, Singh YI, Sanjana RK, Chaudhary N, Saldanha D et al.; Study of extended spectrum beta-lactamases (ESBLs) producing Klebsiella species in various clinical specimens: A preliminary report. Journal of College of Medical Sciences Nepal 2010; 6(3): 19-23.

13. Sahly H., Aucken H., Benedi V.J., Forestier C., Fussing V., Hansen D.S., Ofek I., Podschun R., Sirot D., Tomas J.M., Sandvang D., and Ullmann U. Increased Serum Resistance in Klebsiella pneumonia Strains Producing ExtendedSpectrum beeta-Lactamases. Antimicrob Agents Chemother 2004; 9(48):3477-3482.

14. Shukla I, Tiwari R, Agrawal M; Prevalence of extended spectrum $\beta$ lactamse producing Klebsiella pneumoniae in tertiary care hospital. Indian Journal Medical Microbiology 2004; 22(2): 87-91.

15. Ali Abdel Rahim KA, Ali Mohamed AM; Prevalence of extended spectrum $\beta$ lactamase producing Klebsiella pneumoniae in clinical isolates. Jundishapur Journal of Microbiology 2014; 7(11): e17114.

16. Kitara LD, Anywar AD, Acullu D, Odongo-Aginya E, Aloyo J, and Fendu M, Afr Health Sci. 2011; (11): S34-S39. 